\title{
The Computation of $\boldsymbol{R}_{0}$ for Discrete-Time Epidemic Models with Dynamic Heterogeneity
}

\author{
M. C. M. DE JONG \\ CDI-DLO, Lelystad, The Netherlands \\ O. DIEKMANN \\ CWI, Amsterdam, The Netherlands, and Institute of Theoretical Biology, \\ Leiden, The Netherlands \\ AND \\ J. A. P. HEESTERBEEK \\ CWI, Amsterdam, The Netherlands
}

Received 26 January 1993; revised 1 April 1993

\begin{abstract}
An explicit algorithm is given for the computation of the basic reproduction ratio $R_{0}$ (or the net reproduction ratio $R$ in the case of a not wholly susceptible population) for a class of discrete-time epidemic models. These models allow for a finite number of different individual types, type changes at fixed type-dependent intervals, arbitrary contact intensity between individuals of the various types, and variable infectivity. The models reflect the situation where an infectious disease spreads in a population of animals that are reared in different stables on farms.

In addition, it is shown analytically that the reproduction ratio depends, for any given type, on the product of the susceptibility and the total infectivity of that type and not on these factors separately. We call this product the transmission weight of the type. The maximum overall transmission weight gives an upper bound for the reproduction ratio, irrespective of the particular submodels for type change and contact structure. Reduction of all transmission weights below 1 , by vaccination or some other control measure, will result in $R<1$ and will hence lead to eradication of the disease.
\end{abstract}

\section{INTRODUCTION}

Imagine a viral infection in a heterogeneous population of animals that are reared in different stables on farms and are moved from one stable to another (and possibly also between farms) at regular intervals. Suppose one wants to study the possible spread of the viral infection in such a situation or evaluate possibilities for eradication of an already 
established infection. It is common practice in handling problems of this kind to study the basic reproduction ratio $R_{0}$ (see, e.g., [2], [5]). $R_{0}$ is the expected number of new infections caused by a typical infected individual during its entire infectious period in a virgin (i.e., completely susceptible) population that is in a stable demographic steady state at the moment the infection is introduced.* For any eradication strategy to be effective, it has to reduce the net reproduction ratio $R$ of the infective agent in the non-virgin controlled population below 1 .

In this paper we present an algorithm to compute $R_{0}$ (or $R$ as the case may be) as a function of relevant and measurable parameters that govern the spread of the virus from individual to individual and from stable to stable. We do this for a broad class of multigroup models where the type (group) of an individual can be dynamic, where we can specify an arbitrary contact structure between individuals of the various types, and where the infectivity is allowed to be a function of the time elapsed since infection took place. We are concerned with calculation of $R_{0}$ for this class of models and the dependence of $R_{0}$ on the ingredients of the model.

In [10] it was shown, in a general abstract setting, that $R_{0}$ for heterogeneous populations is defined as the dominant eigenvalue of the so-called next-generation operator. This operator, which in the case of a finite number of different individual types takes the form of a matrix, describes, on a generation basis, the expected number of new cases that a certain type of newly infected individual causes and how these new cases are distributed over all possible individual types at the moment infection takes place.

In order to determine the elements of the next-generation matrix, one has to specify submodels for various processes, one of which is change of type. The "type" can be static or dynamic, and in the latter case one has to provide an adequate description of the dynamics. A popular assumption is that the per capita probability per unit of time that a type change will occur is a (type-dependent) constant. This Markovian point of view ("type" equals "state" at the individual level) has the technical advantage that it leads to ordinary differential equations when describing the deterministic (law of large numbers) limit at the population level (see, e.g., [15]). It implies that the sojourn time

\footnotetext{
* One can argue whether or not a new symbol should be introduced in the case of a population that is not wholly susceptible because some control measures have been applied. One could denote the corresponding net reproduction ratio by $R$. However, from a mathematical point of view, $R_{0}$ and $R$ are reproduction ratios calculated in precisely the same manner, the only difference being that the virgin and controlled populations differ in their steadv-state structure at the moment of invasion.
} 
distributions for the various states are exponential. In the context of animal farms with transport among stables, the sojourn time is usually the same for all animals, and thus the distribution is far from exponential.

We can incorporate sojourn times with nonexponential distributions by adopting the semi-Markovian point of view that the per capita probability per unit of time that a type change will occur depends not only on the type but also on the type-age. In the present paper we restrict our attention to the relatively simple (and therefore parameterscarce!) case where the sojourn time is determined by two independent mechanisms: death, with a constant probability per unit of time, and "transport" from one type to another after preset type-dependent time intervals. Furthermore, we take into account variable infectivity as a function of the time that has elapsed since infection took place (infection-age). For models of this complexity, it is neither easy nor helpful to give the next-generation matrix explicitly. However, we can determine the entries of this matrix directly from our ingredients with an efficient algorithm.

Section 2 is devoted to the basic ingredients of the model and describes the relation of these ingredients to measurable quantities. In Section 3 we given an annotated algorithm to compute the entries of the next-generation matrix, and we show analytically what the influence is on the next-generation matrix and $R_{0}$ of some specific changes in the ingredients. In Section 4 we illustrate our approach with some examples based on the spread of Aujeszky's disease virus (ADV) of pigs on a farrow-to-finish farm.

\section{THE INGREDIENTS OF THE MODEL}

\subsection{DEMOGRAPHY}

We distinguish individuals according to their type, in which we combine both physiological differences (e.g., age and being pregnant) and spatial differences (i.e., the spatial compartment where the individual currently resides). The current physiological state determines the susceptibility of an individual (probability that the individual becomes infected for a given level of infectious material in its direct surroundings), and the physiological state at the moment of becoming infected determines the production of infectious material (output) as a function of the time that has passed since the individual became infected. The spatial structure determines who "meets" whom. We represent both structures with a single label taking values in $\{1,2, \ldots, n\}$.

We want to allow that both the physiological and spatial characteristics of a given individual change over time. Type alone does not suffice 
to calculate the probability of being of a certain type at future times; we need in addition to consider the type-age of the separate individuals. We measure this type-age in discrete steps, and for an individual of type $i$ it takes the values $\left\{1,2, \ldots, \sigma_{i}\right\}$. We will often loosely speak of "age" instead of "type-age" and refer to the combination $(i, a)$ of type $i$ and type-age $a$ as the state of the individual.

We assume that if an individual becomes of a given type $i$, it starts with age 1 and changes type at age $\sigma_{i}$, as long as it does not die along the way. We postulate an age-independent survival probability $\pi_{i}$ per time step for individuals of type $i$. Let $\pi_{i} k_{j i}$ denote the probability that an individual that is in age class $\sigma_{i}$ of type $i$ will be in age class 1 of type $j$ one time step later. So the matrix $K=\left(k_{i j}\right)_{1 \leqslant i, j \leqslant n}$ describes the redistribution of types.

We allow for the immigration of $w_{i}$ individuals in age class 1 of type $i$ at each time step. The stable type distribution with influx can then be calculated as follows. Suppose there are $s_{i}$ individuals in age class 1 of type $i$ at each time step; then $s_{i} \pi_{i}^{\sigma_{i}}$ individuals leave type $i$ alive at each time step. Hence, we have the consistency requirement

$$
s=w+K D s,
$$

where $D=\operatorname{diag}\left(\pi_{i}^{\sigma_{i}}\right)$ is the diagonal matrix with entries $\pi_{i}^{\sigma_{i}}$. We obtain

$$
s=(I-K D)^{-1} w,
$$

and we conclude that the stable demographic steady state, in the absence of infection, is given by $s_{i} \pi_{i}^{a-1}$ for types $i \in\{1,2, \ldots, n\}$ and type-ages $a \in\left\{1,2, \ldots, \sigma_{i}\right\}$. The total number of individuals of type $i$ in the demographic equilibrium is therefore

$$
s_{i}\left(1+\pi_{i}+\cdots+\pi_{i}^{\sigma_{i}-1}\right)
$$

In the situation described so far, the matrix $K D$ should have dominant eigenvalue less than 1 , and a steady demographic state results from a continuous influx $w$ of new individuals from the outside. The situation where a steady demographic state results from reproduction within the system, balanced by outflow (as incorporated in the matrix $K$ ), can be described as follows. Assume that reproduction necessarly coincides with a change of type (this can always be achieved by an appropriate definition of the types, see, e.g., Section 4). The vector $D s$ describes the (constant) outflow in a steady demographic state. Let the matrix $B$ describe reproduction. More precisely, let $b_{i j}$ denote the expected 
number of individuals of type $i$ produced by an individual that reaches the maximal age of type $j$. Then consistency requires that

$$
s=B D s+K D s
$$

or, in other words, that the matrix $(B+K) D$ has dominant eigenvalue 1 and that $s$ is the corresponding eigenvector. In the concrete example of the management of a farm, we can consider $B$ and $D$ as given, to some extent, by nature, and then $K$ has to be adjusted to satisfy the eigenvalue condition (again we refer to Section 4 for an example).

In Section 2.2 we make special assumptions that guarantee that the $s_{i}$ characterizing the demographic steady-state drop out of the expressions necessary to calculate $R_{0}$.

\subsection{THE INFECTION PROCESS}

When, during a certain time interval, an individual of type $j$ with type-age $a$ becomes infected, then we colloquially speak of $(j, a)$ as the state at "birth" of the individual ("born" with respect to the infection). During the next time interval the infection-age of this individual is 1 , and its state is either $(j, a+1)$ (whenever $\left.a<\sigma_{j}\right)$ or $(l, 1)$ for some $l$ with $k_{l j}>0$ whenever $a=\sigma_{j}$. We neglect the possibility that a newly infected individual dies during the same time interval in which it becomes infected. However, we do take removal during the change in type into account, so if $\sum_{l=1}^{n} k_{l j}<1$, then it is possible that an individual is born although it will not live to reach infection-age 1 .

If the individual is born with type $j$, then we can calculate the probability that its state at birth is $(j, a)$. Since all individuals of type $j$ are equally susceptible and experience the same force of infection, this probability is given by

$$
\frac{s_{j} \pi_{j}^{a-1}}{\sum_{k^{j}=1}^{\sigma_{j}} s_{j} \pi_{j}^{k-1}}=\frac{\pi_{j}^{a-1}}{\sum_{k=1}^{\sigma_{j}} \pi_{j}^{k-1}}=\frac{\pi_{j}^{a-1}-\pi_{j}^{a}}{1-\pi_{j}^{\sigma_{j}}}
$$

when $0<\pi_{j}<1$, whereas the probability is $1 / \sigma_{j}$ for $\pi_{j}=1$.

We assume that the product $h_{\beta} f_{j}$ is a measure for the expected amount of infectious material shed by an individual of birth type $j$ with infection-age $\beta$ given that the individual is not removed by diseaseunrelated causes. (Removal by disease-unrelated causes will be incorporated in the quantities $e_{l j}$ to be defined below.) For $h_{\beta}$ we assume that its support is given by some (discrete) interval $\left[\theta_{-}, \theta_{+}\right]$, and we normalize $\sum_{\beta=\theta_{-}}^{\theta_{+}} h_{\beta}=1$. The factor $f_{j}$ reflects possible differences in infectivity that are birth-type-related. So, note that the way in which infectivity 
depends on infection-age is, by assumption, the same for all types; the influence of type is at most a scaling factor.

Remember that differences in type include differences in the spatial compartment in which the individual resides. We assume that the type of an individual fully determines the contact intensity. More precisely, we postulate that a fraction $c_{i j}$ of the infectious material shed by individuals of type $j$ contributes to the force of infection acting on individuals of type $i$.

In addition to differences in infectivity $\left(f_{j}\right)$, we allow for differences in susceptibility. We define type $i$ susceptibility $g_{i}$ as follows. The probability that an individual of type $i$ becomes infected when exposed for a period of length $\Delta t$ to an intensity (a concentration) of infectious material $\zeta$ is declared to be $1-e^{-\zeta g_{i} \Delta t}$. Provided that $\zeta \Delta t$ is small, it is legitimate to replace $1-e^{-\zeta g_{i} \Delta t}$ by its linearization $\zeta g_{i} \Delta t$, and we will do so in all that follows. The limit this sets to the period $\Delta t$ depends on the value of $\zeta$. In this paper we concentrate on the early stages of an epidemic, when $\zeta$ is small and therefore $\Delta t$ is not severely restricted. In addition we adopt the point of view that if one unit of infectious material is released into a group of $N$ animals, the probability that this unit will reach a particular individual within the time step $\Delta t$ is $1 / N$. Moreover, when several types are present in the same compartment, the probability per time step that a unit of infectious material reaches a particular individual of type $i$ is

$$
\frac{N_{i}}{\sum N_{j}}\left(\frac{1}{N_{i}}\right)
$$

where the summation is over all the different types within the compartment and where $N_{i}=s_{i}\left(1+\cdots+\pi_{i}^{\sigma_{i}-1}\right)$ is the population size of type $i$ individuals in the demographic steady state. The first factor is just the fraction of type $i$ individuals in the compartment, and we shall include this factor in the contact matrix $C$ defined above. As a consequence of these assumptions, the probability that any member of the type $i$ individuals will become infected when they as a group come in contact with one unit of infectivity is

$$
\frac{g_{i}}{s_{i}\left(1+\pi_{i}+\cdots+\pi_{i}^{\sigma_{i}-1}\right)}
$$

In our model, the factors $f_{j}$ and $g_{i}$ enter the next-generation matrix only as a product. Therefore, the calculations in Section 3 have straightforward generalizations to the case where these products are replaced by the more general factors. However, in most applications it seems 
reasonable to assume that infectivity and susceptibility are separate multiplicative factors.

\section{AN ALGORITHM}

To calculate $R_{0}$ we consider a newly infected individual born with type $j$. Define numbers $m_{i j}$ as the expected number of secondary cases that an individual born with type $j$ will produce among the individuals with type $i$. The matrix $M=\left(m_{i j}\right)_{1 \leqslant i, j \leqslant n}$ is the next-generation matrix in the sense of [10], and $R_{0}$ is the dominant eigenvalue of $M$.

In the algorithm described below we concentrate on the computation of the matrix components $m_{i j}$ from the ingredients of the model, that is, the number of types $n$, the vector of maximal type-ages $\sigma$, the survival vector $\pi$, the type redistribution matrix $K$, the "mixing" matrix $C$, the infectivity and susceptibility vectors $f$ and $g$, and finally the normalized vector $h$ describing the fraction of infectivity produced as a function of infection-age. One can then use standard numerical software to determine the dominant eigenvalue of the matrix $M$.

We consider an infected individual that was born with type $j$ (we will refer to this individual as the index case for convenience). As time proceeds, the type of the index case will change, given that the individual does not die. Define $f_{j} e_{l j}, j \in\{1,2, \ldots, n\}$, as the total amount of infectivity that the index case is expected to produce while it is of type $l$. Note that it is possible that $\sum_{l=1}^{n} e_{l j}<1$ because now we do not take removal by disease-unrelated causes into account.

The component $m_{i j}$ of $M$, that is, the expected number of victims the index case makes among the susceptibles of type $i$, is then given by (remember the last expression from Section 2)

$$
m_{i j}=\frac{s_{i}\left(1+\pi_{i}+\cdots+\pi_{i}^{\sigma_{i}-1}\right) g_{i}}{s_{i}\left(1+\pi_{i}+\cdots+\pi_{i}^{\sigma_{i}-1}\right)} f_{j} \sum_{l=1}^{n} c_{i l} e_{l j}=g_{i} f_{j} \sum_{l=1}^{n} c_{i l} e_{l j} .
$$

The task remains to give an algorithm for the computation of the coefficients $e_{l j}$.

At first we ignore that the index case will contribute to $e_{j j}$ before it changes its type for the first time after becoming infected. We consider the birth type of an infected individual as the root of a treelike graph and all possible types as vertices that may be passed through as time proceeds. From the root, a finite number of main branches will grow to new vertices, one branch for each type $l$ with $k_{l j}>0$. These branches will, in turn, split into secondary branches, tertiary branches, etc. The idea of the algorithm is to systematically, and economically, walk along all branches that emanate from the root, and every time we pass 
through a vertex $l \in\{1,2, \ldots, n\}$, "update" the relevant coefficient $e_{l j}$. In the end, all coefficients $e_{l j}, l \in\{1,2, \ldots, n\}$ will have been computed simultaneously. We carry out this computation for all birth types $j \in$ $\{1,2, \ldots, n\}$.

The individual contributions to a given coefficient $e_{l j}$ will depend on the infection-age of the index case at the time it (re)enters type $l$, because the infectivity $h$ is a function of infection-age. Define $E(l, \alpha)$ as the expected fraction of infectivity produced by an individual of infection-age $\alpha$ and state $(l, 1)$ during the entire connected period in which this individual is of type $l$. Our assumptions imply that

$$
E(l, \alpha)=\sum_{\beta=\max \left\{\alpha, \theta_{-}\right\}}^{\min \left\{\alpha+\sigma_{l}-1, \theta_{+}\right\}} h_{\beta} \pi_{l}^{\beta-\alpha}
$$

(here and in the following we use the convention that a sum equals zero whenever the upper index bound is less than the lower index bound).

Consider an individual born with state $(j, b)$. The conditional probability that the individual leaves type $j$ alive (as far as non-infectionrelated causes of death are concerned; infection-related causes are incorporated in $h$ ), given that it is alive at the end of the time interval in which its type-age is $b$, is $\pi_{j}^{\sigma_{j}} / \pi_{j}^{b}=\pi_{j}^{\sigma_{j}-b}$. The infection-age of this individual is $\sigma_{j}-b$ in the last time interval in which it is of type $j$, and it is $\sigma_{j}-b+1$ in the first time interval in which the individual is, possibly, of another type.

Now consider an individual born with type $j$. The probability that such an individual has type-age $b$ at the moment of infection and leaves type $j$ alive is

$$
\frac{\pi_{j}^{b-1}}{\sum_{k=1}^{\sigma_{j}} \pi_{j}^{k-1}} \pi_{j}^{\sigma_{j}-b}=\frac{\pi_{j}^{\sigma_{j}-1}}{\sum_{k=1}^{\sigma_{j}} \pi_{j}^{k-1}}=: \phi_{j} \pi_{j}^{\sigma_{j}}
$$

which is independent of $b$ ! So, if we consider all possibilities for the type-age at birth, we obtain a constant output in number, while the infection-age at type-age 1 of the next type varies from 1 (when $b=\sigma_{j}$ ) to $\sigma_{j}$ (when $b=1$ ). So, for example, if the next type is $l$, this yields a contribution

$$
\pi_{j}^{\sigma_{j}} \phi_{j} \sum_{\alpha=1}^{\sigma_{j}} E(l, \alpha)
$$

to the fraction of infectivity shed while of type $l$. When we follow this 
individual further, we get contributions

$$
\pi_{j}^{\sigma_{j}} \phi_{j} \sum_{\alpha=T+1}^{T+\sigma_{j}} E(l, \alpha)
$$

to the fraction of infectivity shed while of type $l$, where $T$ is the sum of the type durations of all types the individual passed through after the initial $j$ and preceding the present type $l$ (given that it stays alive). Note that the factor $\phi_{j}$ is the same all the time, so we can save some computational effort by delaying the multiplication by $\phi_{j}$ until the end of our computations. The factor $\pi_{j}^{\sigma_{j}}$ will be incorporated in the variable $p$ (see below).

From our systematic tree walk, we use two procedures that will be called upon time and again in the main program: procedure $S^{+}$(which takes a step to the right in our tour of the horizontal tree) and procedure $S^{-}$(which starts by taking a step to the left). Let $Q$ be an array of integers of variable length corresponding to the types that are visited by the index case after it becomes infected and the order in which they are visited (i.e., the route of the index case through the tree). Let the variable $T$ denote the total duration of all type periods in $Q$ except the first and the last, and let $p$ denote the product of (1) the probability, given that the index case leaves its birth type alive, that the particular route through type space described by $Q$ is actually followed and the individual stays alive, and (2) the factor $\pi_{j}^{\sigma_{j}}$ (this last factor is incorporated in order to have a unified description of $S^{+}$for the first and all subsequent steps). With $Q:=Q \oplus\left\{q_{r}\right\}$ we denote the addition of a new vertex type at the right-hand end to the existing array $Q$, and with $Q:=Q \ominus\left\{q_{r-1}\right\}$ we denote the removal of the last vertex type on the right side of $Q$.

Suppose the current status of the route $Q$ by our index case is $Q=\left[q_{0}, q_{1}, \ldots, q_{r-1}, q_{r}\right]$ for $r \geqslant 1, q_{i} \in\{1,2, \ldots, n\}$. The root of the tree, that is, the birth type of the index case, is $y_{0}$. The procedure $S^{+}(x)$ adds the vertex $q_{r+1}=x$ to the route, updates the variables $p$ and $T$ for "living through" the type $x$, and updates the coefficient $e_{x q_{0}}$.

Procedure $S^{+}(x)$

$$
\begin{aligned}
q_{r+1} & :=x \\
p & :=k_{q_{r+1} q_{r}} \pi_{q_{r}}^{\sigma_{q_{r}} p} \\
e_{q_{r+1} q_{0}} & :=e_{q_{r+1} q_{0}}+p \sum_{\alpha+\sigma_{q_{0}}} E\left(q_{r+1}, \alpha\right) \\
T & :=T+\sigma_{q_{r+1}} \\
Q & :=Q \oplus\left\{q_{r+1}\right\}
\end{aligned}
$$

end procedure $S^{+}$. 
The addition of new vertex types at the right end of $Q$ can stop for two reasons. First it may be that at some point a vertex $i$ is added from which no new branches can emanate because $k_{l i}=0$ for all $l \in$ $\{1,2, \ldots, n\}$. Second, we stop adding new elements when the total time $T$ of the route $Q$ is greater than the maximum time $\theta_{+}$during which infectivity can be emitted. From the point of view of the disease, we are not interested in the ramifications of the particular branch of the tree that may lie beyond a vertex where the infectious period elapses. In both of the "stop situations" we retrace our steps, removing vertex types from the right end of $Q$, simultaneously backdating the variables $T$ and $p$ appropriately, until an element of $Q$ is reached from which progress into another branch of the tree is possible. This is the content of procedure $S^{-}$. As an auxiliary tool we need the procedure Next. The procedure $\operatorname{Next}(r, s)$ determines the lowest possible type index that can follow type index $r$, subject to the constraint that the next type index must be greater than or equal to $s$. When there actually is no next type index possible satisfying the constraint, Next is put, somewhat arbitrarily, equal to $n+1$.

Procedure $\operatorname{Next}(r, s)$

$$
\begin{aligned}
& \text { if } s=n+1 \text { then Next }:=n+1 \\
& \text { else if } k_{s r}>0 \text { then Next }:=s \\
& \text { else } N \operatorname{ext}(r, s+1)
\end{aligned}
$$

end procedure Next.

The current status of the route is taken as $Q=\left[q_{0}, q_{1}, \ldots, q_{r-1}, q_{r}\right]$ for $r \geqslant 1$.

\section{Procedure $S^{-}$}

$$
\begin{aligned}
T & :=T-\sigma_{q_{r}} \\
p & :=p / k_{q_{r} q_{r-1}} \pi_{q_{r-1}}^{\sigma_{q_{r-1}}} \\
x & :=\operatorname{Next}\left(q_{r-1}, q_{r}+1\right) \\
Q & :=Q \ominus\left\{q_{r}\right\} \\
& \text { If } x<n+1 \text { then } S^{+}(x) \\
& \text { else } \\
& \text { if not } Q=\left[q_{0}\right] \text { then } S^{-}
\end{aligned}
$$

end procedure $S^{-}$.

Finally, we are ready for the main algorithm, which is by now easy to formulate. We just walk through the trees we obtain by taking in turn each possible birth type as root. At each vertex we choose, using Next, the lowest type following the present type for which progress is possible. 
We have to take care of two loose ends. We have to multiply all coefficients $e_{l j}$ with the survival factor $\phi_{j}$, and the contribution that the index case with birth type $j$ makes to the infectivity toward susceptibles of type $j$, before the first postinfection type change occurs, still has to be taken into account.

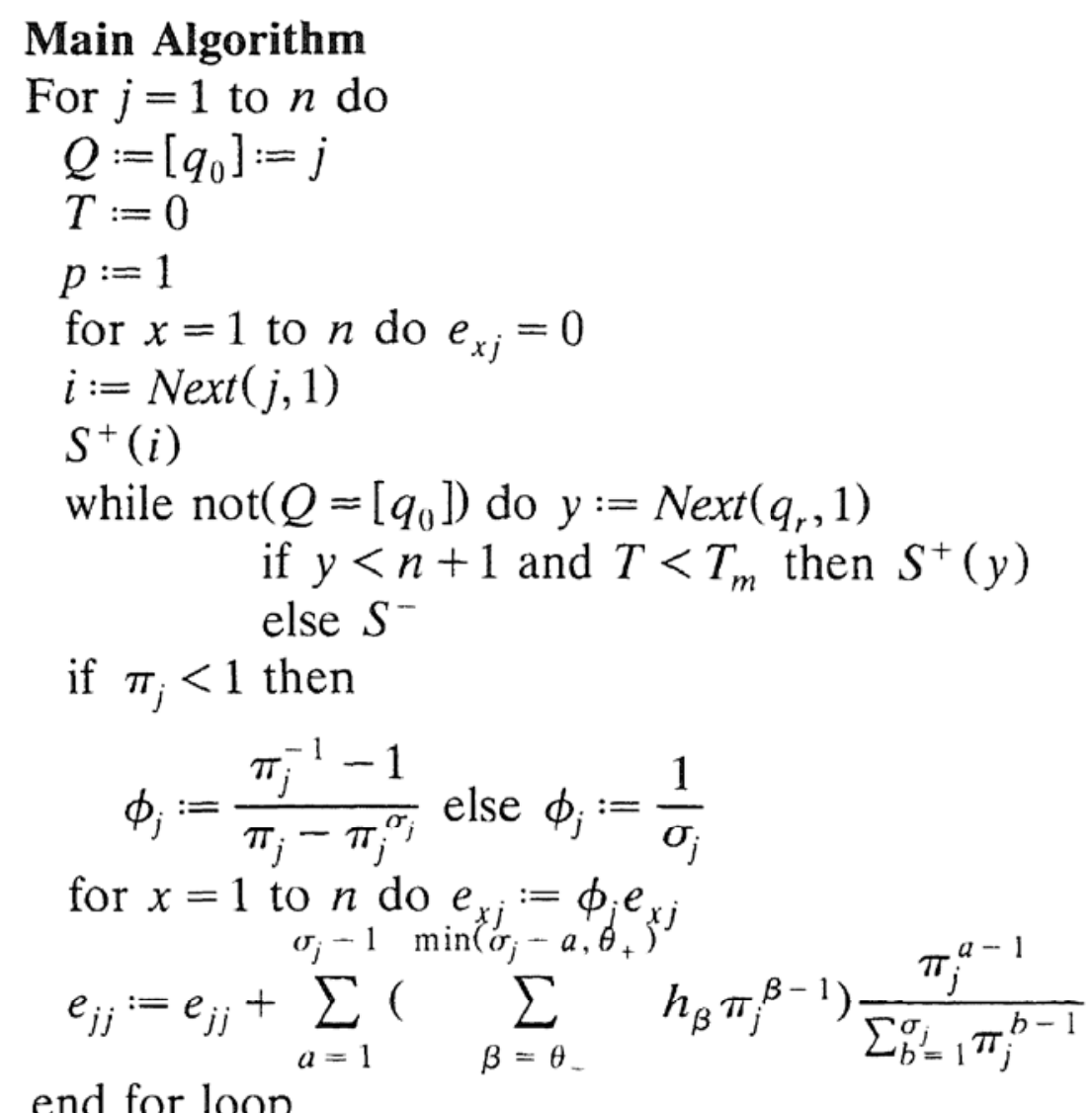

end for loop

We end this section by deriving two analytic results about $R_{0}$ in the present framework. The idea that these results should hold was born after experimenting under various biological assumptions with a computer implementation of the algorithm.

We note from Equation (1) that $R_{0}$ for a homogeneous population consisting of a single type $i$ is equal to the product of the infectivity $f_{i}$ and the susceptibility $g_{i}$. To avoid confusion with the $R_{0}$ for the heterogeneous population consisting of more than one type, we refer to the product $f_{i} g_{i}$ as the transmission weight of type $i$. One can now rightfully wonder whether different partitionings of the two factors within the product would influence $R_{0}$. The following easy observation shows that this is not the case.

LEMMA 1

Define $f_{i}^{\prime}=\left(1 / \kappa_{i}\right) f_{i}$ and $g_{i}^{\prime}=\kappa_{i} g_{i}$, for $\kappa_{i}>0$ and $i \in\{1, \ldots, n\}$. Consider matrices $M$ and $M^{\prime}$ with the entries given by (1) with appropriate 
placing of accents for $M^{\prime}$. Then the dominant eigenvalue of $M$ is equal to the dominant eigenvalue of $M$.

Proof. The eigenvalue problems of $M$ and $M^{\prime}$ are related in the following way: $M x=\lambda x \Leftrightarrow M^{\prime} y=\lambda y$ where $y_{i}=\kappa_{i} x_{i}, i \in\{1, \ldots, n\}$. Therefore, the eigenvalues of $M$ and $M^{\prime}$ are the same, and hence the dominant eigenvalues are the same.

Knowing that $R_{0}$ depends on the products $f_{i} g_{i}$, we now derive an estimate for $R_{0}$ in terms of these transmission weights.

\section{THEOREM 1}

For the matrix $M$ with entries given by (1), the dominant eigenvalue is less than or equal to $\max _{1 \leqslant i \leqslant n}\left\{f_{i} g_{i}\right\}$.

Proof. We define $g_{i}^{\prime}=1$ and $f_{i}^{\prime}=f_{i} g_{i}$ for $i \in\{1, \ldots, n\}$. Let a matrix $M^{\prime}$ be defined by (1) with $f_{i}^{\prime}$ and $g_{i}^{\prime}$ as infectivity and susceptibility of type $i, i \in\{1, \ldots, n\}: m_{i j}^{\prime}=f_{j}^{\prime} \sum_{l=1}^{n} c_{i l} e_{l j}$. Then, by Lemma 1 , the eigenvalues of $M^{\prime}$ are equal to the eigenvalues of $M$. From the definitions of $e_{i j}$ and $c_{i j}$ we know that $\sum_{i=1}^{n} e_{i j} \leqslant 1$ for all $j \in\{1, \ldots, n\}$ and $\sum_{i=1}^{n} c_{i j}=1$ for all $j \in\{1, \ldots, n\}$. A well-known upper estimate for the dominant eigenvalue $\lambda_{d}$ of a positive matrix (see, e.g., [14]) is, when applied to $M^{\prime}$,

$$
\lambda_{d} \leqslant \max _{1 \leqslant j \leqslant n} \sum_{i=1}^{n} m_{i j}^{\prime} .
$$

This leads to

$$
\begin{aligned}
\lambda_{d} & \leqslant \max _{1 \leqslant j \leqslant n} f_{j}^{\prime} \sum_{i=1}^{n} \sum_{l=1}^{n} c_{i l} e_{l j} \\
& =\max _{1 \leqslant j \leqslant n} f_{j}^{\prime} \sum_{l=1}^{n} e_{l j} \sum_{i=1}^{n} c_{i l} \\
& \leqslant \max _{1 \leqslant j \leqslant n} f_{j}^{\prime}=\max _{1 \leqslant j \leqslant n} f_{j} g_{j} .
\end{aligned}
$$

\section{AUJESZKY'S VIRUS DISEASE ON A PIG BREEDING FARM}

On a farrow-to-finish farm (see, e.g., [11]), sows are kept to produce piglets, and these piglets are reared to a certain weight and then sold. Some of the piglets are reared to serve as replacement for the sows in the production cycle. We consider four types of individuals (see Figure 1 ): (1) pregnant sows, all in one stable, (2) sows that have farrowed in the nursery stable, (3) suckling piglets in the nursery stable, and (4) piglets in the rearing stage (finishing pigs) in a third stable. The pregnant sows have a gestation time of 17 weeks ( $\sigma_{1}=170$ units) and a 


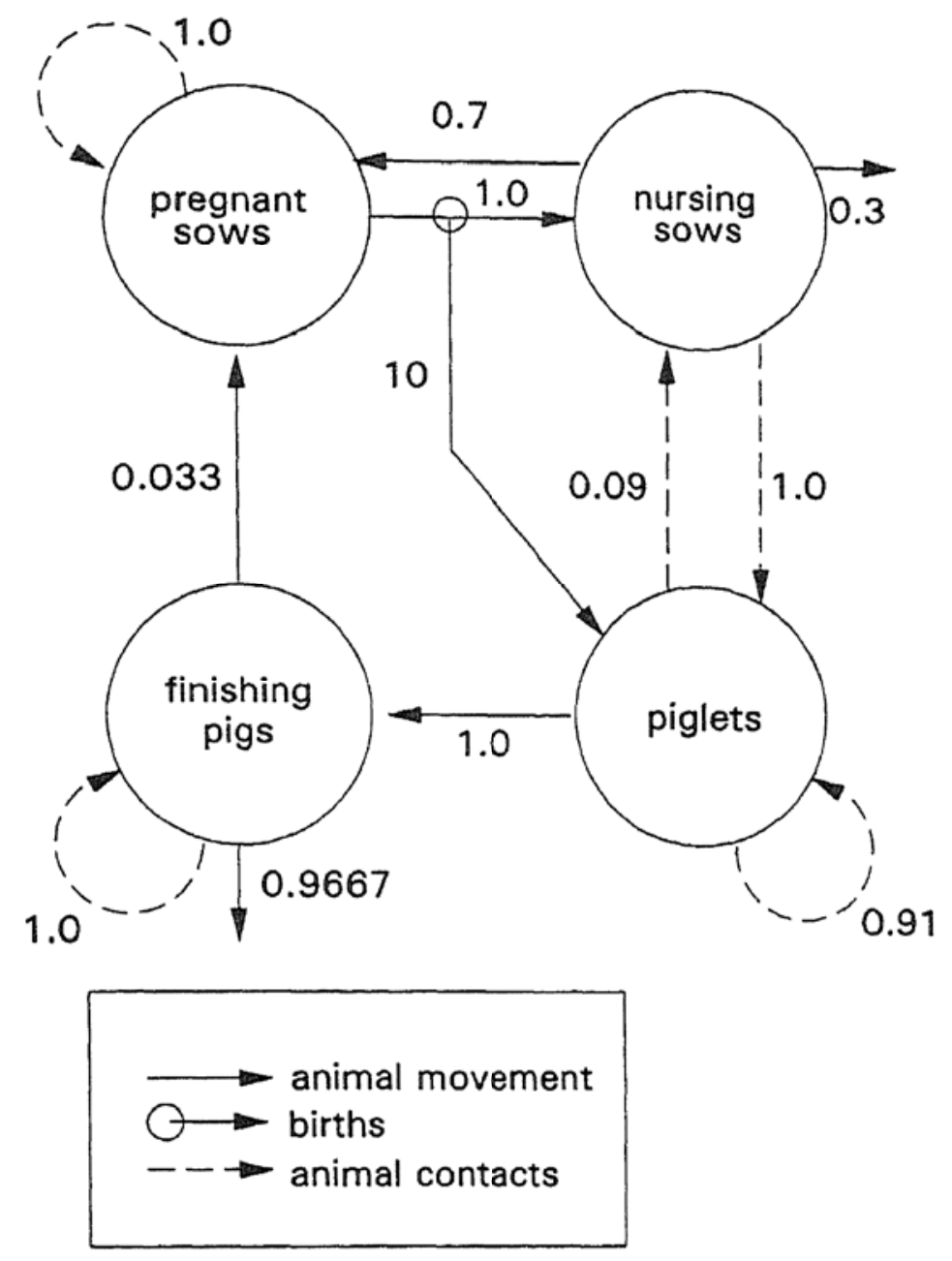

FIG. 1.

nursing phase of 5 weeks ( $\sigma_{2}=\sigma_{3}=50$ units). The rearing phase of the piglets lasts 30 weeks ( $\sigma_{4}=300$ units). Each sow is assumed to have 10 piglets, and therefore $91 \%$ of the individuals in the nursery stable are piglets and $9 \%$ are sows (the sows mainly have contacts with their own piglets). Upon leaving the nursery unit, $70 \%$ of the sows are impregnated again and returned to the farrowing unit for a new cycle. All the gilts, after leaving the nursery unit, are reared in the finishing unit to let them mature. After maturation, a fraction of these pigs will enter the production cycle.

The above assumptions lead to the following direct animal-to-animal contact matrix $C$,

$$
C=\left(\begin{array}{llll}
1 & 0 & 0 & 0 \\
0 & 0 & 0.09 & 0 \\
0 & 1 & 0.91 & 0 \\
0 & 0 & 0 & 1
\end{array}\right)
$$


The demography of these farms is controlled by the farmer. He or she sells animals in such a way that the population size remains constant. The above description leads to the following animal transport matrix $K$,

$$
K=\left(\begin{array}{cccc}
0 & 0.7 & 0 & k_{14} \\
1 & 0 & 0 & 0 \\
0 & 0 & 0 & 0 \\
0 & 0 & 1 & 0
\end{array}\right)
$$

The survival probability per time step is assumed to be 1 for all types except the piglets of type 3 , for which mortality is assumed to be $10 \%$ over the total nursing period $\left(\pi_{j}=0\right.$ for $j \in\{1,2,4\}$ and $\pi_{3}=0.9979$ per unit). New animals enter only through births $\left(b_{31}=10\right.$ per unit, and $b_{i j}=0$ for $i \neq 3, j \neq 1$ ).

For our example we assume that the fraction of matured gilts from the finishing unit that enter the production cycle $\left(k_{14}\right)$ is the variable that is used by the farmer as a means to control the population size. It is easy to check that for

$$
k_{14}=\frac{1-k_{12} k_{21} d_{11} d_{22}}{k_{43} b_{31} d_{11} d_{33} d_{44}}
$$

the dominant eigenvalue of the matrix $B D+K D$ (see Section 2.1) is 1 . This leads to $k_{14}=0.033$ for the parameter values chosen above.

The ingredients specified up to now determine the demography of the pig herd. Now consider infection with $\mathrm{ADV}$ in this herd. Information about ADV in pigs is available from experimental infection of individual pigs with and without vaccination $[8,9]$ and from transmission experiments with and without vaccination [6]. The duration of the infectious period is approximately 2 weeks ( 20 units). We assume a constant infectivity during each of these 20 units: $h_{\beta}=0.05$ for $\beta \in$ $\{1,2, \ldots, 20\}$. In the transmission experiments of De Jong and Kimman [6], $R$ was estimated in homogeneous groups, both vaccinated and unvaccinated, of animals of a single type. The quantity so estimated is the transmission weight of the particular type used in the experiment. From the experiments it was estimated that for vaccinated pigs of type 4 the transmission weight is 0.5 , while for unvaccinated pigs of the same type it is 23 .

Accurate information about infectivity and susceptibility separately is not available. It is possible to measure virus excretion in pigs by determining the virus content of saliva samples. Unfortunately, this measure is highly variable [8], and in addition it is unclear how much virus reaches other individuals. One could measure susceptibility based 
on the inverse of the dose needed to infect $50 \%$ of the individuals [9]. Again, accurate measures cannot be obtained easily; it would require the use of too many experimental animals. However, as we have seen in Lemma 1 in Section 3, it does not matter for $R$ how precisely the transmission weights are proportioned into infectivity and susceptibility.

Let us first assume that all types of individuals have transmission weight equal to the experimentally estimated weight for piglets in the rearing stage (type 4). One possible parameterization for a totally vaccinated population is $f_{i}=1, g_{i}=0.5$ for $i \in\{1,2,3,4\}$. The next-generation matrix $M$, calculated by implementing the algorithm from Section 3 in TurboPascal, is

$$
\left(\begin{array}{llll}
0.47 & 0.07 & 0.00 & 0.00 \\
0.00 & 0.00 & 0.04 & 0.00 \\
0.03 & 0.40 & 0.36 & 0.00 \\
0.00 & 0.00 & 0.10 & 0.48
\end{array}\right)
$$

Calculation of the dominant eigenvalue of $M$ leads to $R=0.48$. By Theorem 1 in Section 3, $R$ cannot exceed the maximum of the transmission weights. The reason $R$ is smaller in the present case is that some infectious animals are removed from the population before they can emit their total infectivity. To illustrate the effect of a different parameterization, we assume that nursing sows are much more infective $\left(f_{2}=\right.$ $\left.10.0, g_{2}=0.05\right)$ and suckling piglets are much more susceptible $\left(g_{3}=\right.$ $\left.20.0, f_{3}=0.025\right)$, keeping the transmission weights constant. The resulting $R$ is the same by Lemma 1 , but the next-generation matrix $M$ has changed considerably to

$$
\left(\begin{array}{cccc}
0.47 & 0.74 & 0.00 & 0.00 \\
0.00 & 0.00 & 0.00 & 0.00 \\
1.24 & 158.0 & 0.35 & 0.00 \\
0.00 & 0.00 & 0.10 & 0.48
\end{array}\right)
$$

We know from observations that it is possible that ADV does spread among vaccinated pigs on farrow-to-finish farms. In the light of Theorem 1, one explanation for this is that the transmission weight of some type is greater than 1 . This could well be the case for the piglets (type 3 ). These individuals are in practice not vaccinated but only have protection from maternal antibodies. Let us assume that these piglets are totally unprotected against virus transmission, although they can possibly be protected against the occurrence of clinical signs. Under this assumption, the transmission weight of these piglets is 23 (estimated 
from the experiments mentioned before). The next-generation matrix is, by parameterizing as $f_{3}=1$ and $g_{3}=23$,

$$
\left(\begin{array}{rcrr}
0.47 & 0.07 & 0.00 & 0.00 \\
0.00 & 0.00 & 0.00 & 0.00 \\
1.42 & 18.17 & 16.19 & 0.00 \\
0.00 & 0.00 & 0.10 & 0.48
\end{array}\right)
$$

and the resulting $R=16.23$.

Transmission among the piglets is just one explanation for virus circulation in a vaccinated population; other explanations are possible. However, because of Theorem 1 there are restrictions: whenever transmission from one type of animal to another type of animal is the product of infectivity of one type and susceptibility of the other type, there must be some type of individual in the population for which the transmission weight is greater than 1 .

\section{DISCUSSION}

When formulating multigroup models of epidemic spread, one has to specify the migration of individuals (or transport, depending on the context) from group to group as well as the intensity of contacts within groups and between groups. In addition, one has to specify the death, removal, and recruitment processes that collectively determine demographic turnover.

Given such a specification, the next-generation matrix and its dominant eigenvalue $R_{0}$ are well defined. Yet it may be far from easy to compute the entries of the next-generation matrix from the ingredients of the model specification. In this paper we have presented an algorithm for such a computation for a fairly general class of model specifications, particularly relevant to viral diseases spreading on animal farms but possibly applicable in completely different contexts as well.

How relevant is group structure for the analysis of the invasion/elimination problem? If contact intensity is highly variable among groups, a so-called core group can bring $R_{0}$ above 1 whereas it would be below 1 if all individuals had the average contact intensity (see [12], [13]). In a similar spirit we have shown in Section 4 that a high susceptibility of unvaccinated piglets can have a tremendous influence on $R$ of the overall population. Thus it appears that averaging has to be done by means of the next-generation matrix and not by taking averages of individual properties with respect to population composition (see [1] for a general monotonicity result). Consider the final example from Section 4, where $f_{i}=1, i \in\{1,2,3,4\}$, and $g_{i}=0.5, i \in\{1,2,4\}$, but with a high susceptibility of piglets $g_{3}=23$. Straightforward averaging of the 
transmission weights as an approximation to $R_{0}$, keeping account of the fact that type 2 and type 3 animals reside in the same stable, leads to

$$
\frac{1}{4}\left(0.5+\frac{1}{11} 0.5+\frac{10}{11} 23+0.5\right)=5.5,
$$

whereas we know from Section 4 that the dominant eigenvalue of the next-generation matrix for this particular case is the (much) higher 16.2 (which again illustrates Adler's result in [1]).

When comparing different members of a family of models with each other, it is far from trivial how one should gauge them, and in fact normalization may be quite important for the results one obtains. In this paper, type $i$ individuals are characterized by a susceptibility parameter $g_{i}$ and an infectivity parameter $f_{i}$. In Lemma 1 we showed that $R_{0}$ depends on the products $f_{i} g_{i}$, which we baptised "transmission weights," and not on the factors separately, while in Theorem 1 we established that $R_{0}$ is bounded from the above by the maximum of $f_{i} g_{i}$. This inequality depends heavily on our normalization, which guarantees that the number of contacts an individual is expected to have does not depend on its migratory path (as embodied in the matrices $K$ and $C$ ), as long as it is not removed by death or otherwise. In the context of sexually transmitted diseases such a normalization is usual (see, e.g., [13]), but in other families of models, contacts outside one's own group are simply added to those within the group, and then of course $R_{0}$ for the overall population may well be substantially bigger than the maximum of the within-group $R_{0}$ 's (note that one can interpret $f_{i} g_{i}$ as such, should one wish to do so). See [3] or [10] for this phenomenon.

From a practical point of view, a simple upper bound for $R$ is quite effective since it combines nicely with the inequality $R<1$ that decides about the success of a control strategy. Here "simple" means that it is based on a few parameters, the transmission weights only. For Aujeszky's disease virus among pigs, the transmission weights are currently being estimated experimentally (cf. De Jong and Kimman [6]). The experimental work also serves to check one of the basic assumptions of this paper, that the per capita number of contacts depends on population density but not on population size in terms of numbers (so if a farm is twice as large, both in the number of animals and in the size of the stables, then the per capita number of contacts remain the same; see De Jong et al. [7] and Bouma et al. [4]).

We believe that a combination of observational work in the field, experimental work in the laboratory, and the development of theoretical and computational tools as in this paper will lead in the long run to a much better understanding and management of viral diseases spreading on animal farms. 


\section{REFERENCES}

1 F. Adler, The effects of averaging on the basic reproduction ratio, Math. Biosci. 111:89-98 (1992).

2 R. M. Anderson and R. M. May, Infectious Diseases of Humans: Dynamics and Control, Oxford Univ. Press, New York, 1991.

3 V. Andreasen and F. B. Christiansen, Persistence of an infectious disease in a subdivided population, Math. Biosci. 96:239-253 (1989).

4 A. Bouma, M. C. M. De Jong, and T. G. Kimman, Experimental support for one type of mass-action formulation in epidemiology, in preparation.

5 M. C. M. De Jong and O. Diekmann, A method to calculate, for computersimulated infections, the threshold value $R_{0}$ that predicts whether or not the infection will spread, Prev. Vet. Med. 12:269-285 (1992).

6 M. C. M. De Jong and T. G. Kimman, Experimental quantification of vaccineinduced reduction in virus transmission, submitted to Vaccine.

7 M. C. M. De Jong, O. Diekmann, and J. A. P. Heesterbeek, How does transmission of infection depend on population size?, submitted to Mollison, D. (Ed.) CUP.

8 P. W. De Leeuw and J. T. Van Oirschot, Vaccines against Aujeszky's disease: evaluation of their efficacy under standardized laboratory conditions, Vet. Quart. 7:191-197 (1985).

9 K. De Smet, K. De Waele, and M. Pensaert, Virulent Aujeszky's disease virus infectious dose in vaccinated and non-vaccinated pigs, Proc. IPVS, 65 (1992).

10 O. Diekmann, J. A. P. Heesterbeek, and J. A. J. Metz, On the definition and the computation of the basic reproduction ratio $R_{0}$ in models for infectious diseases in heterogeneous populations, J. Math. Biol. 28:365-382 (1990).

11 B. T. Grenfell and G. Smith, Mathematical model for the impact of a pseudorabies epizootic on the productivity of a farrow-to-finish operation, Am. J. Vet. Res. 51:156-164 (1990).

12 H. W. Hethcote \& J. A. Yorke, Gonorrhea Transmission: Dynamics and Control (Lect. Notes Biomath. 56), Springer-Verlag, Berlin, 1984.

13 J. A. Jacquez, C. P. Simon, and J. S. Koopman, Core groups and the $R_{0}$ 's for subgroups in heterogeneous SIS and SI models, submitted to Mollison, D. (Col.) CUP.

14 M. A. Krasnosel'skij, J. A. Lifshits, and A. V. Sobolev, Positive Linear Systems: The Method of Positive Operators, Heldermann Verlag, Berlin, 1989.

15 D. Mollison, Differentiating diseases, Nature 358:29-30 (1992). 